\title{
Surface ship shock modeling and simulation: two-dimensional analysis
}

\author{
Young S. Shin and Leonard D. Santiago \\ Department of Mechanical Engineering, Naval \\ Postgraduate School, Monterey, CA 93943, USA \\ Received 9 September 1997 \\ Revised 11 May 1998
}

The modeling and simulation of the response of a surface ship system to underwater explosion requires an understanding of many different subject areas. These include the process of underwater explosion events, shock wave propagation, explosion gas bubble behavior and bubble-pulse loading, bulk and local cavitation, free surface effect, fluid-structure interaction, and structural dynamics. This paper investigates the effects of fluid-structure interaction and cavitation on the response of a surface ship using USA-NASTRAN-CFA code. First, the one-dimensional Bleich-Sandler model is used to validate the approach, and second, the underwater shock response of a two-dimensional mid-section model of a surface ship is predicted with a surrounding fluid model using a constitutive equation of a bilinear fluid which does not allow transmission of negative pressures.

\section{Introduction and background}

The U.S. Navy has conducted a large number of underwater explosion testing and ship shock trials since World War II. It has become increasingly difficult to perform a full scale naval ship shock trial in the open sea because of severe opposition from the standpoint of environmental protection and rising cost. One solution is to use ship shock modeling and simulation to predict the dynamic behavior of ship systems subjected to underwater explosions.

The response of ship structures subjected to underwater explosion is greatly complicated by the free field problem (e.g., incident/reflected/refracted/rarefection wave propagation, gas bubble oscillation and migration toward free surface, bulk cavitations), complex fluidstructure interaction phenomena, and the dynamic behavior of the structure.
The finite element code used for structural modeling, generation of the equations of motion, storing data blocks of structural mass and stiffness matrices, element connection table, basic grid point data table, etc., is MSC/NASTRAN [8]. Stored data blocks are fed into the USA (Underwater Shock Analysis) code [2,3]. The modeling of the surrounding water media is accomplished by use of the USA code, which is a boundary element code based on the doubly asymptotic approximation (DAA) [6,7]. The DAA approach models the acoustic medium surrounding the structure as a membrane covering the wet surface of the structure. The principal advantage of the DAA is that it models the interaction of the structure with the surrounding acoustic medium in terms of wet-surface response variables only, eliminating the need to discretize the surrounding fluid media. The equivalent fluid forces and masses are applied to the interface boundary nodes. Then, the USA code computes the transient response of a ship system subjected to an acoustic shock wave of arbitrary pressure-profile and underwater explosion.

The USA code coupled with the NASTRAN code is suitable for the solution of a variety of underwater shock problems, but not adequate for the case where the fluid cavitation occurs at the fluid-structure interface. An effective means of including hull cavitation phenomena is to model the surrounding fluid using a bilinear fluid model which does not allow transmission of negative pressures. The CFA (Cavitating Fluid Analyzer) code $[4,5]$ is capable of treating cavitating or non-cavitating acoustic fluid volume elements. CFA is a volume element processor with an acoustic fluid formulation based on the displacement potential.

\section{One-dimensional Bleich-Sandler model}

The one-dimensional Bleich-Sandler cavitation model [1] was first considered and this involved modeling of a floating plate on the surface of a bilinear fluid. The finite element models of both the floating plate and bilinear fluid are shown in Fig. 1. This model was used 
to validate the approach in the USA-NASTRAN-CFA code.

The model consists of one 4-node square steel plate element $\left(1.5^{\prime \prime} \times 1.5^{\prime \prime} \times 1^{\prime \prime}\right.$ thick $)$, and 1008 -node fluid volume elements of $1.5^{\prime \prime} \times 1.5^{\prime \prime} \times 1.5^{\prime \prime}$ size. The total depth of fluid is 150 inches. The charge depth is $1.0 \mathrm{e}+7$ inches centered on the center of the plate, and the corresponding peak pressure is 103 psi. The decay constant is $9.958 \mathrm{e}-4 \mathrm{~s}$.

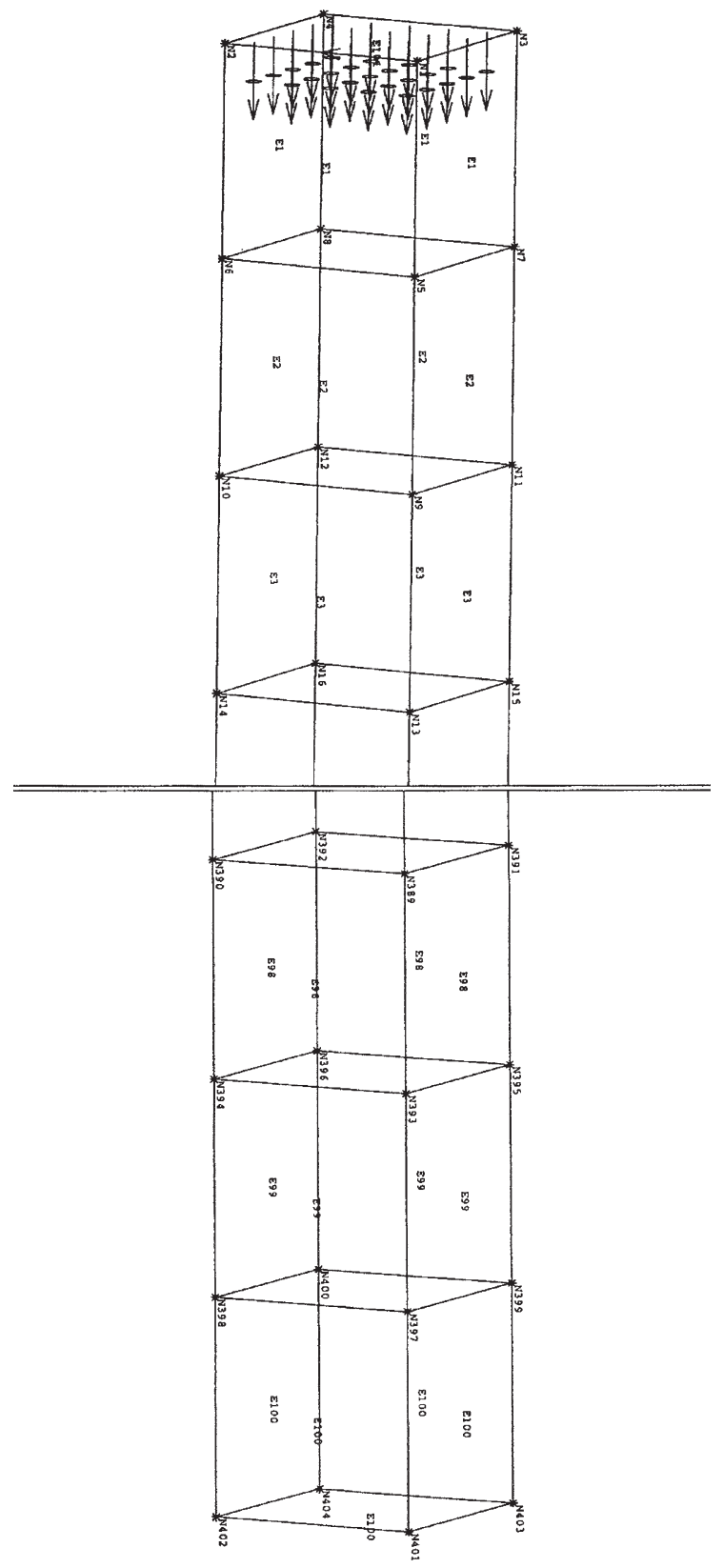

Fig. 1. Bleich-Sandler model.

\section{One-dimensional model analysis results}

Utilizing a time step size of $1.313 \times 10^{-5} \mathrm{~s}, 1200$ time steps were used. With the CFA interface option turned on within the USA code, the computed velocity and pressure at the plate are as shown in Fig. 2. Figure 3 depicts pressures at specific locations along the column of fluid. Because of the numerical computation scheme within the USA code, time 'zero' shown in horizontal axis in each pressure figure refers to the point in time that the incident pressure wave arrives at that particular fluid node and not the global time of plate motion. Total pressure at time zero for each fluid node is the sum of the atmospheric, hydrostatic, and incident pressure wave at that point of arrival from the initial charge location and applied exponential decay. Figure 2 illustrates that the cavitation region does not touch the interface boundary between the fluid and the plate. Node 401 in Fig. 3 confirms the cavitation occurs within the fluid model and does not extend beyond the DAA boundary. Figure 4 depicts the plate velocity and pressure with CFA off.

The calculated time period of zero pressure or cavitation occurrence grows to a period of $0.008 \mathrm{~s}$. halfway through the column and stays approximately the same to the end of the fluid column. In addition, the
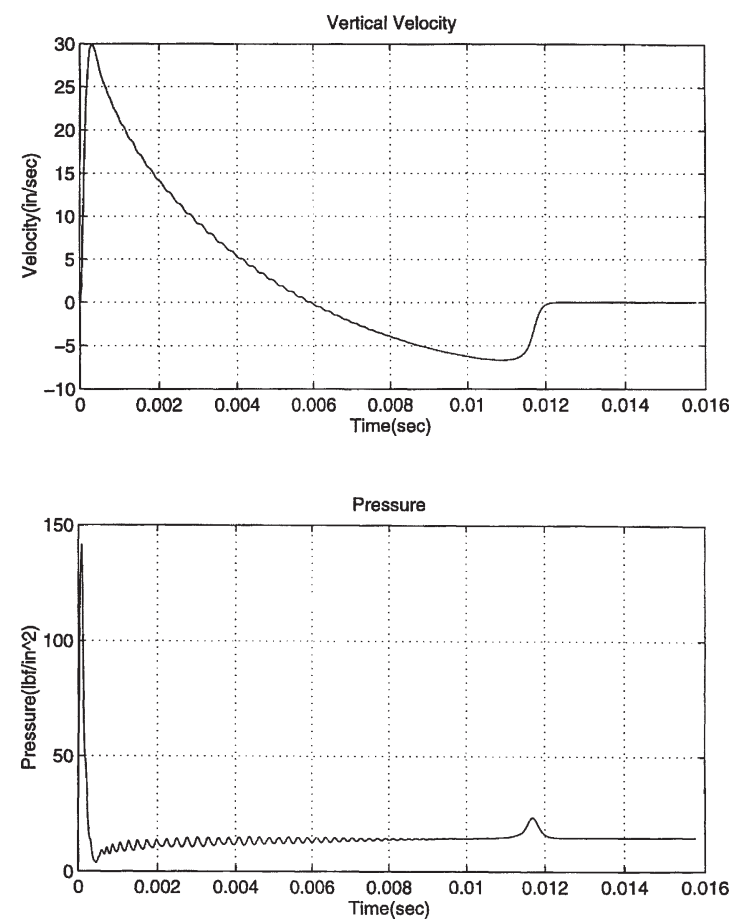

Fig. 2. Bleich-Sandler plate velocity and pressure (CFA on). 

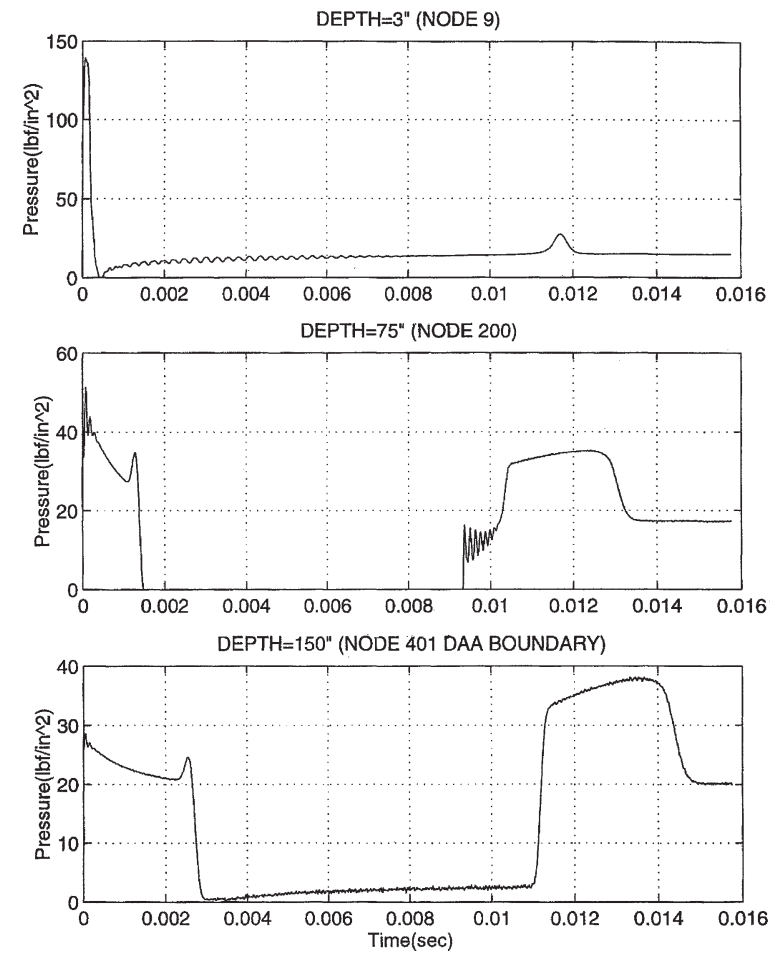

Fig. 3. Fluid pressure at the depth of $3^{\prime \prime}, 75^{\prime \prime}$ and $150^{\prime \prime}$ (DAA boundary) for Bleich-Sandler model (CFA on).
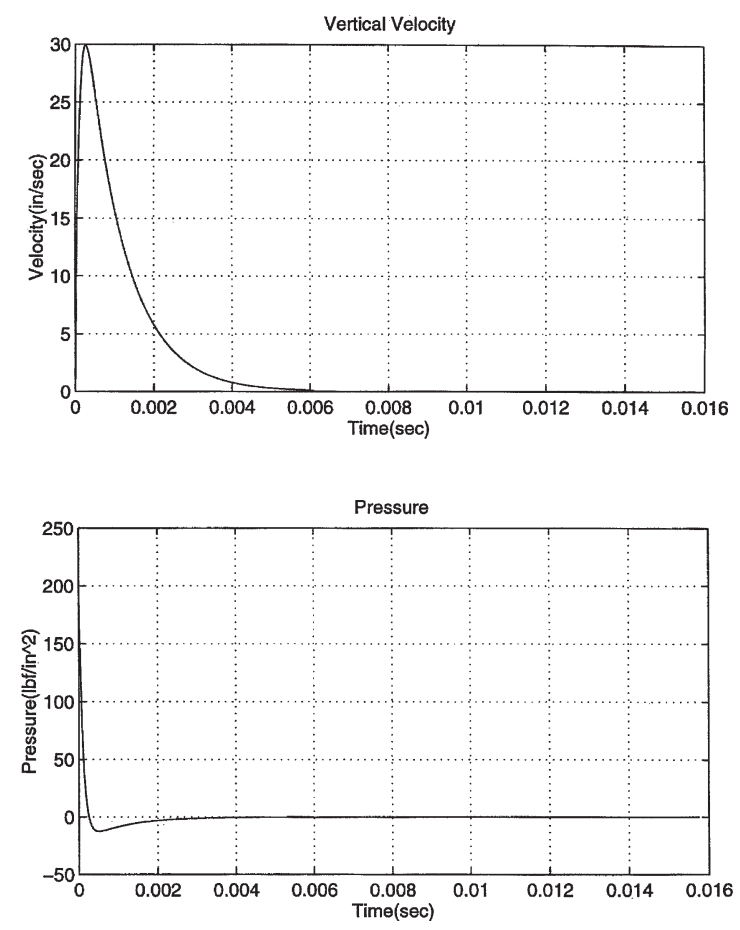

Fig. 4. Bleich-Sandler plate velocity and pressure (CFA off).
CFA captured the cascading effects of cavitation closure which occurred at similar times following incident pressure wave arrivals at each fluid node and closure pressures increased with increasing depth. This closure phenomenon begins at the point $t=0.0108 \mathrm{~s}$ at which the plate experiences an immediate velocity cutoff of the downward direction back to zero by the arrival of this "secondary shock" at the plate. Pressure at this particular time jumps to approximately $14 \%$ of the peak pressure and creates an upward velocity of approximately $16 \%$ which brings the plate velocity back to zero. Multiplying the time by 1004.22 to get it in terms of decay time units of the incident wave and normalizing velocity to $1 / 1000$ of the speed of sound with a sign change due to the $\mathrm{z}$-axis for vertical displacement being defined downward into the fluid, the calculated result matches well with Bleich-Sandler's result as shown in Fig. 5.

\section{Two-dimensional mid-sectional Hull model}

A two-dimensional mid-section of U.S. Navy's Arleigh Burke Destroyer class ship (DDG-51 Flight I) was used, assuming that structural properties remain linear elastic throughout the process. The goal in this 2-D model analysis is to study the responses, specifically peak velocities, with and without cavitation effects, of a surface ship model with a draft of 20 feet. The time frame was limited to the first $30 \mathrm{~ms}$.

Utilizing the USA-NASTRAN-CFA code, Fig. 6 illustrates the location of cross section in the global ship finite element model, 1 foot in width, and Figs 7 to 9 illustrate the details of finite element model. In Figs 7 and 8, both 2-D ship structure and fluid models with dimensions are shown. Figure 9 depicts the outer DAA boundary, free surface, and fluid-structure interface.

The ship structure is steel and model characteristics are as follows:

- number of grid points 452

- number of thin shell elements 230

- number of wet elements 40

- thickness of elements $0.50 \mathrm{inch}$

The CFA elements surrounding the ship hull have the following model characteristics:

- number of fluid nodes 3034

- number of fluid volume elements 1440

- number of DAA boundary face elements 40

- number of free surface face elements 72

- number of face elements contacting hull 40 


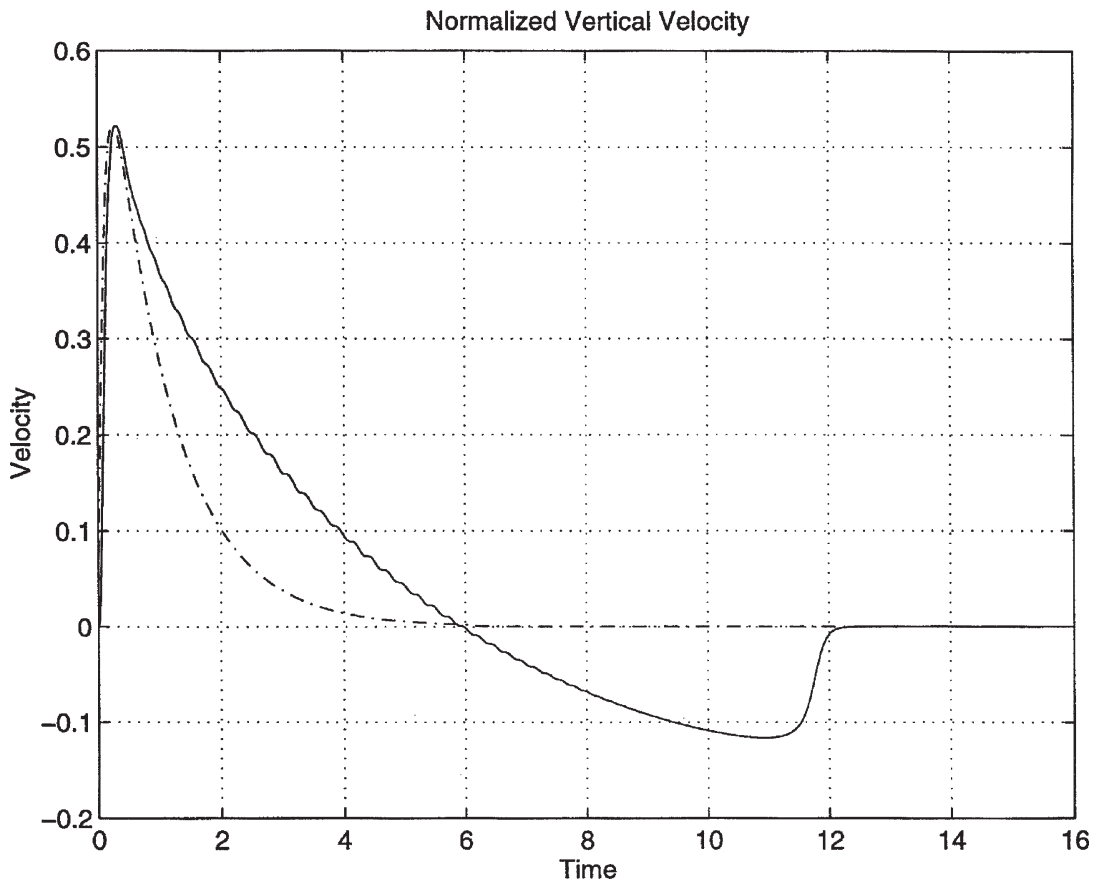

Fig. 5. Normalized vertical velocity for Bleich-Sandler plate (solid Line CFA on; -...- CFA off).

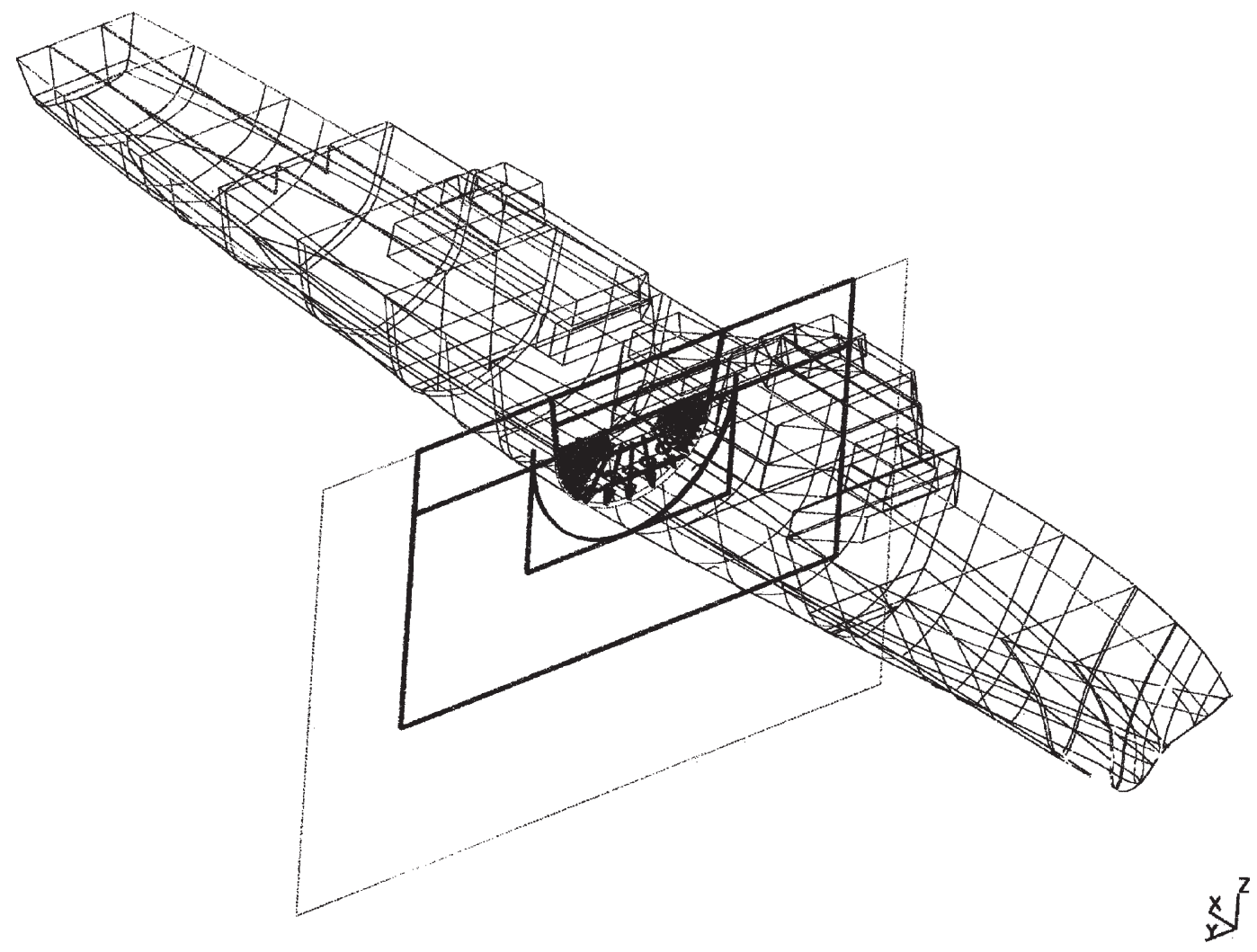

Fig. 6. Two-dimenstional mid-section of U.S. Navy's Arleigh Burke Destroyer class ship (DDG-51 Flight I). 


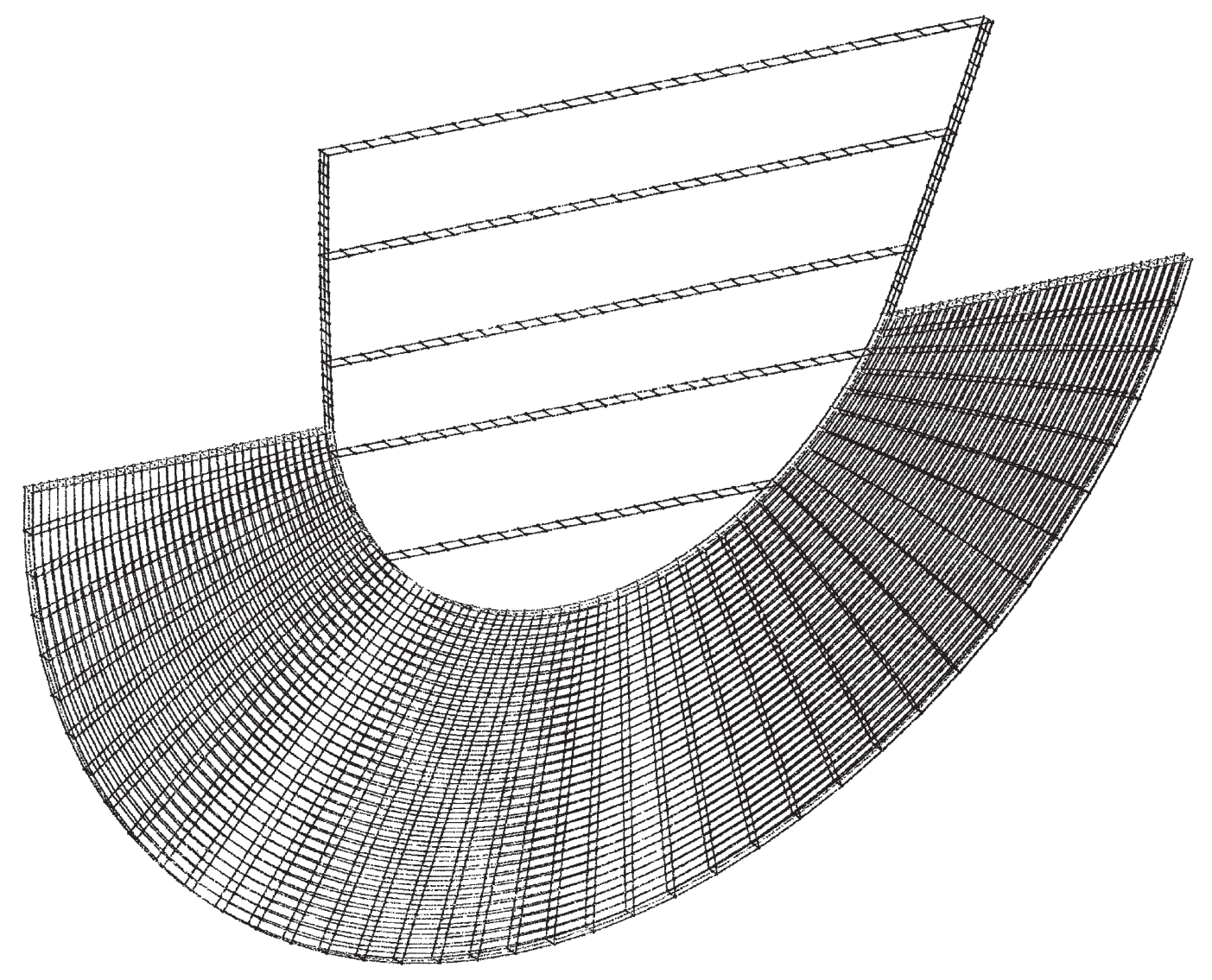

Fig. 7. Two-dimensional ship structure and fluid model.

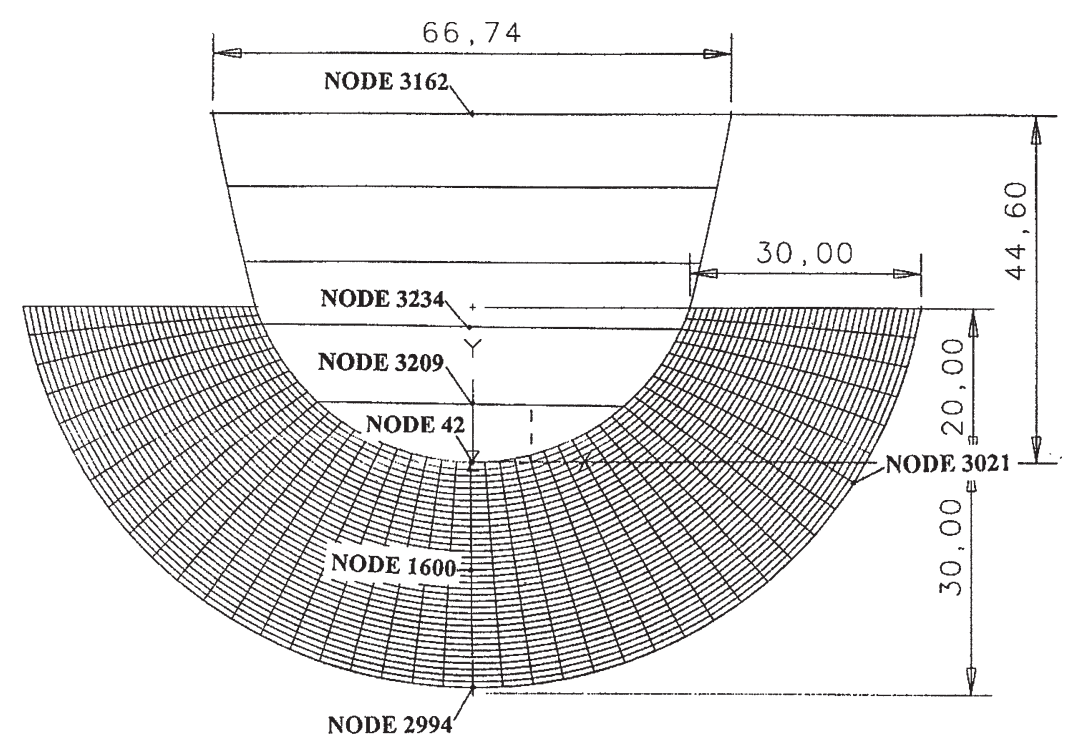

Fig. 8. Node IDs and dimensions of ship structure and fluid model (unit $=$ feet). 


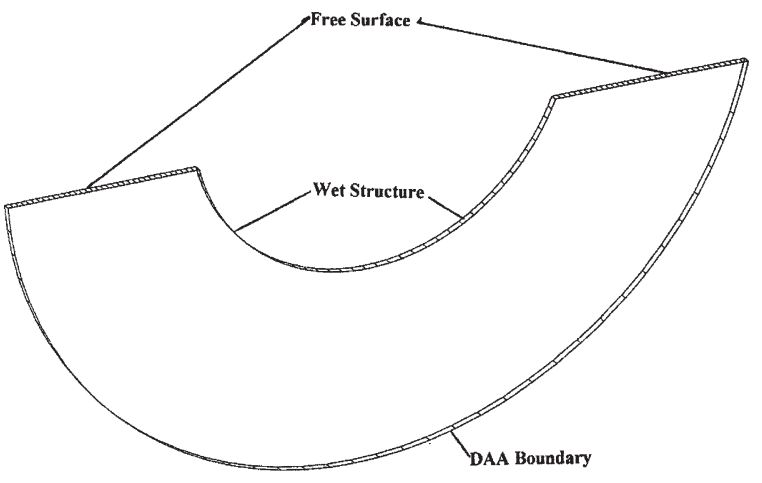

Fig. 9. DAA boundary, free surface and fluid-structure interface.

- maximum number of fluid volume nodes 8

- maximum number of face element nodes 4

A TNT(100 lb) charge is placed at 100 feet below the keel (charge depth along the center of ship cross section is 120 feet).

\section{Two-dimensional model analysis results}

The velocity response at the keel (node 42 shown in Fig. 8) is plotted in Fig. 10(a) through Fig. 10(c) for the first $100 \mathrm{~ms}$. Figure 10(a) shows the cases for "plane wave/CFA-omn" and "plane wave/CFAoff". The comparison shows that the result with CFAon is quite different from that of CFA-off. When the cavitation is turned on, the response shows the high frequency content. Figure 10(b) shows the cases for "DAA/CFA-on" and "DAA/CFA-off". When the CFA is turned off, the DAA boundary is placed at the ship's wet surface of hull. Again, the effect of cavitation is significant. In these two figures, it can also be observed that initial peak velocity is significantly higher in the case when the cavitation is turned off. Figure 10(c) shows the cases for "plane wave/CFA-on" and "DAA/CFA-on". It shows the very interesting result that the two cases are quite close to each other. This result implies that when the cavitation is turned on, the plane shock wave may approximate the response well for early time (here $100 \mathrm{~ms}$ ). However, this may differ at late time.

Figure 11 shows the shock pressure response at the keel. Figure 11(a) is the case for DAA-CFA on and Fig. 11(b) for DAA-CFA off. Figure 11(a) indicates that the hull cavitation occurs immediately after the incident shock wave arrives at the target. It also shows the cavitation closure pulse occurring at a much earlier

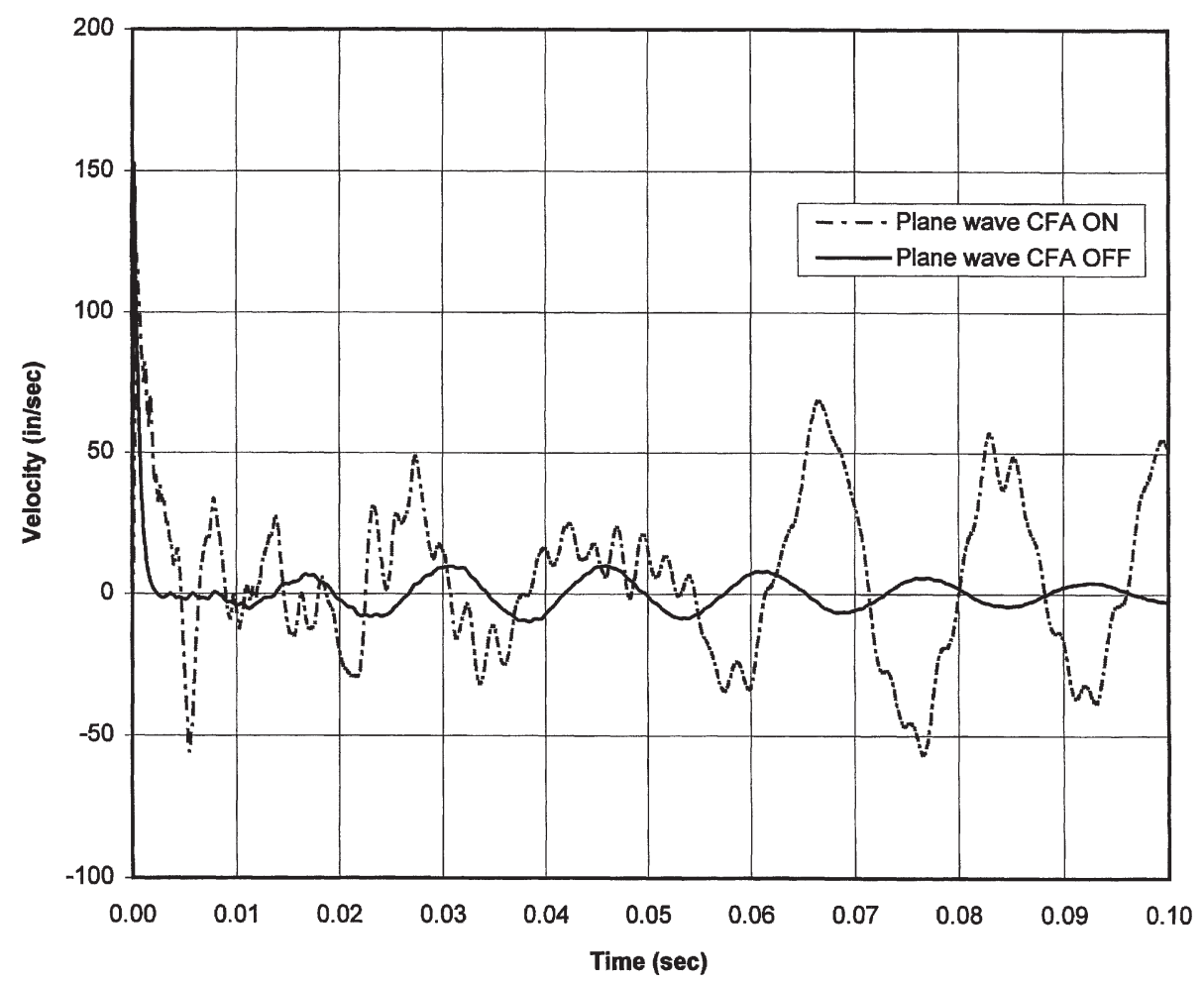

Fig. 10(a). Velocity response at the keel (node 42) for two cases: plane wave/CFA-on, and plane wave/CFA-off. 


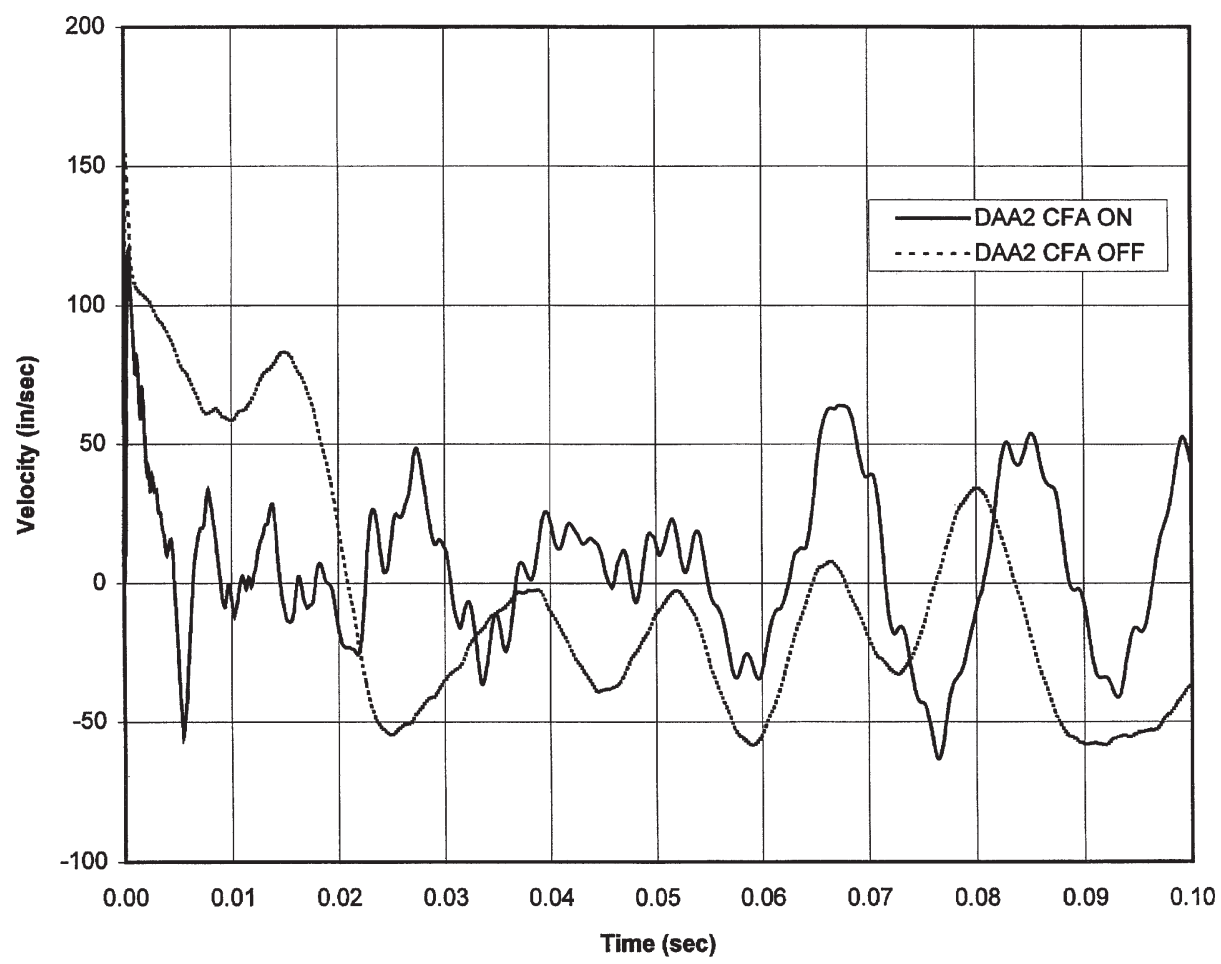

Fig. 10(b). Velocity response at the keel (node 42) for two cases: DAA/CFA-on and DAA/CFA-off.

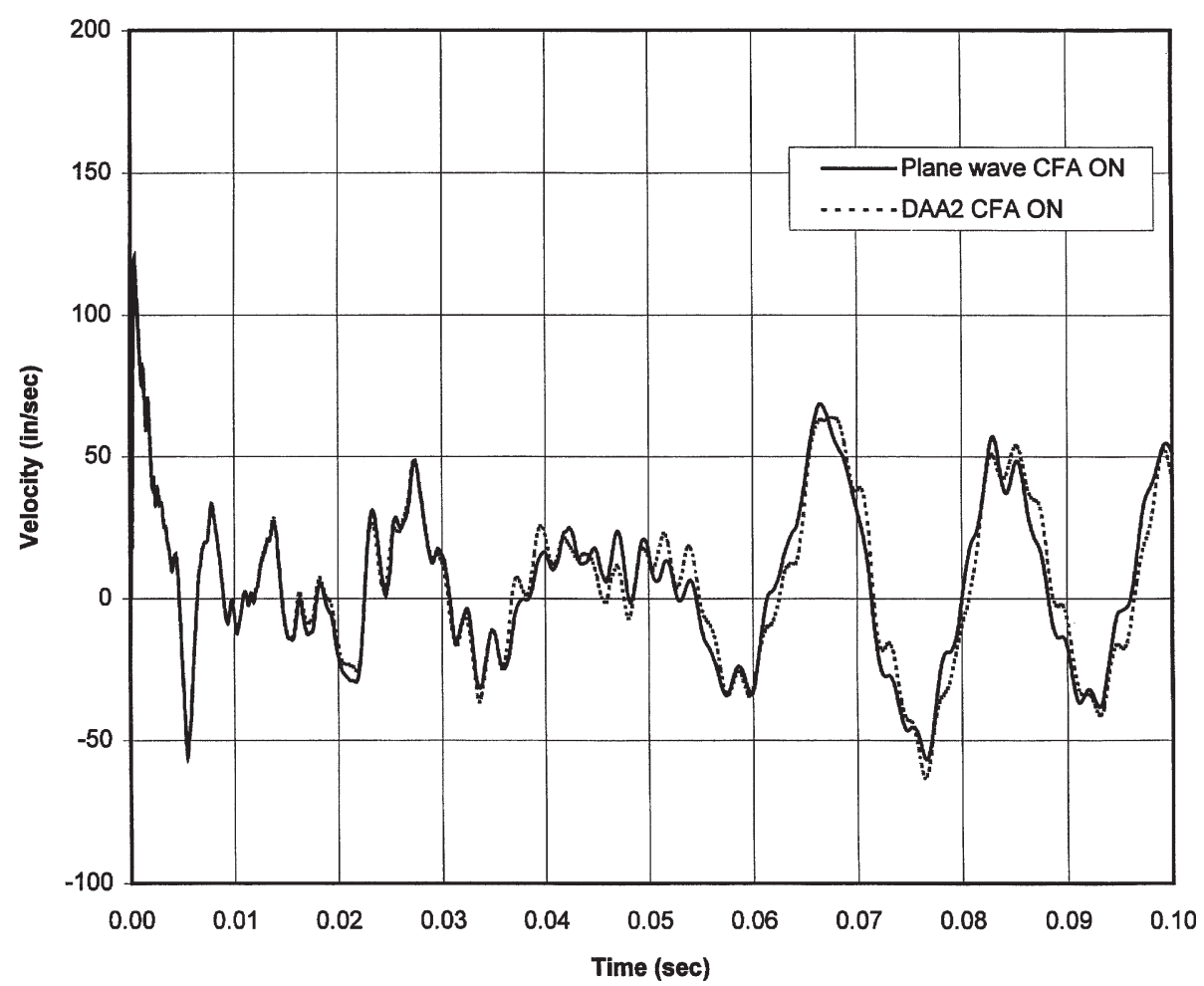

Fig. 10(c). Velocity response at the keel (node 42) for two cases: plane wave/CFA-on and DAA/CFA-off. 


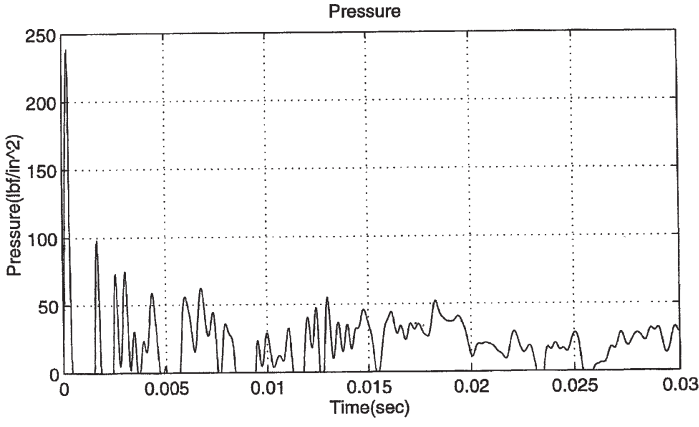

(a) DAA/CFA-ON

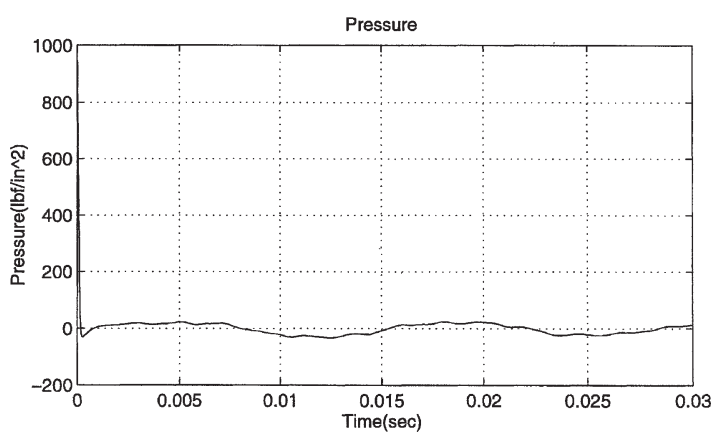

(b) DAAICFA-OFF

Fig. 11. Fluid pressure response at the keel (node 42): (a) DAA/CFA-on and (b) DAA/CFA-off.
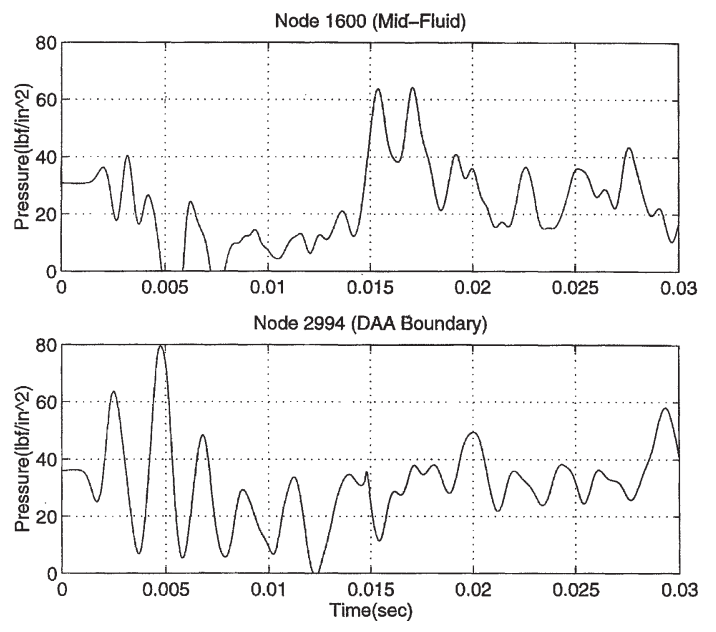

Fig. 12. Fluid pressure responses at the middle and bottom of CFA volume elements

time compared to the Bleich-Sandler case with an appreciably larger pressure peak, $41 \%$ of the initial pressure wave. When the CFA is turned off, extremely high incident shock pressures at early time and small pressure variation at late time are observed as shown in Fig. 11(b).
The pressure responses at the middle and bottom of CFA volume elements (nodes 1600 and 2994 in Fig. 8) are computed and are shown in Fig. 12. It is observed that the middle of CFA volume elements is cavitated in early time and the outskirt of CFA elements is not cavitated.

\section{Summary and conclusions}

It is demonstrated that the whole surface ship shock simulation can be achieved by modeling the ship structure and surrounding fluid using the USA-NASTRANCFA code. Among the many parameters, free surface, fluid-structure interaction and bulk/hull cavitation effects can be easily incorporated in the simulation based analysis.

The studies conducted in this paper have clearly indicated that the cavitation effect must be included in the ship shock simulation and that the cavitation volume must be large enough so that the cavitation boundary does not extend outside the DAA boundary.

The analysis results have shown that cavitation occurs immediately after the incident shock wave hits the target. A significant cavitation closure pressure pulse is also observed in the analysis. The case with no cavitation effect produces sharp and extremely high pressure at the fluid-structure boundary, and also an observed negative pressure. When the cavitation is turned on, the case with plane shock wave may approximate the response well for the early time (here $100 \mathrm{~ms}$ ). Of course, it may differ at late times.

\section{References}

[1] H.G. Bleich and I.S. Sandler, Interaction between structures and bilinear fluids, Int. J. Solids and Structures 6 (1970), 617-638.

[2] J.A. DeRuntz, Jr., T.L. Geers and C.A. Felippa, The underwater shock analysis code (USA-version 3) - reference manual. (1980). DNA Report 5615F.

[3] J.A. DeRuntz, Jr., The Underwater shock analysis code and its applications, in: Proc. 60 Shock and Vibration Symposium, Vol. I, (1989a), pp. 89-107.

[4] J.A. DeRuntz, Jr. and C.C. Rankin, Applications of the USASTAGS-CFA code to nonlinear fluid-structure interaction problems in underwater shock of submerged structures, in: Proc. 60 Shock and Vibration Symposium, Vol. I, (1989b) pp. 121-138.

[5] C.A. Felippa and J.A. DeRuntz, Finite element analysis of shock-induced Hull cavitation, Computer Methods in Applied Mechanics and Engineering 44 (1984), 297-337.

[6] T.L. Geers, Residual potential and approximation methods for three-dimensional fluid-structure interaction problems, J. Acoustic Society of America 49 (1971), 1505-1510. 
[7] T.L. Geers, Doubly asymptotic approximations for transient motions of submerged structures, J. Acoustic Society of America 64 (1978), 1500-1508.
[8] MSC (The MacNeal-Schwendler Corporation), MSC/NASTRAN Quick Reference Guide, Version 67 (1993). 

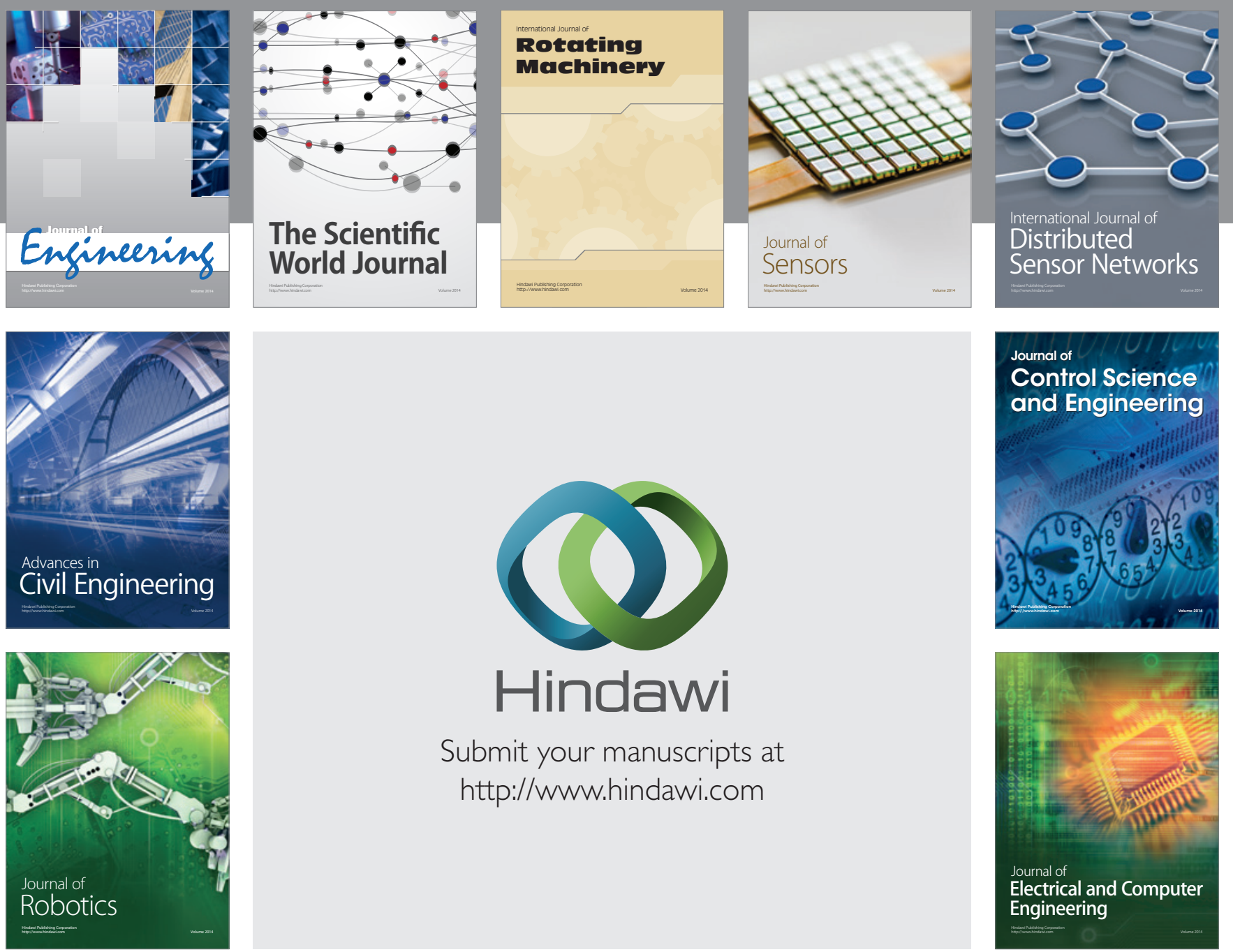

Submit your manuscripts at

http://www.hindawi.com
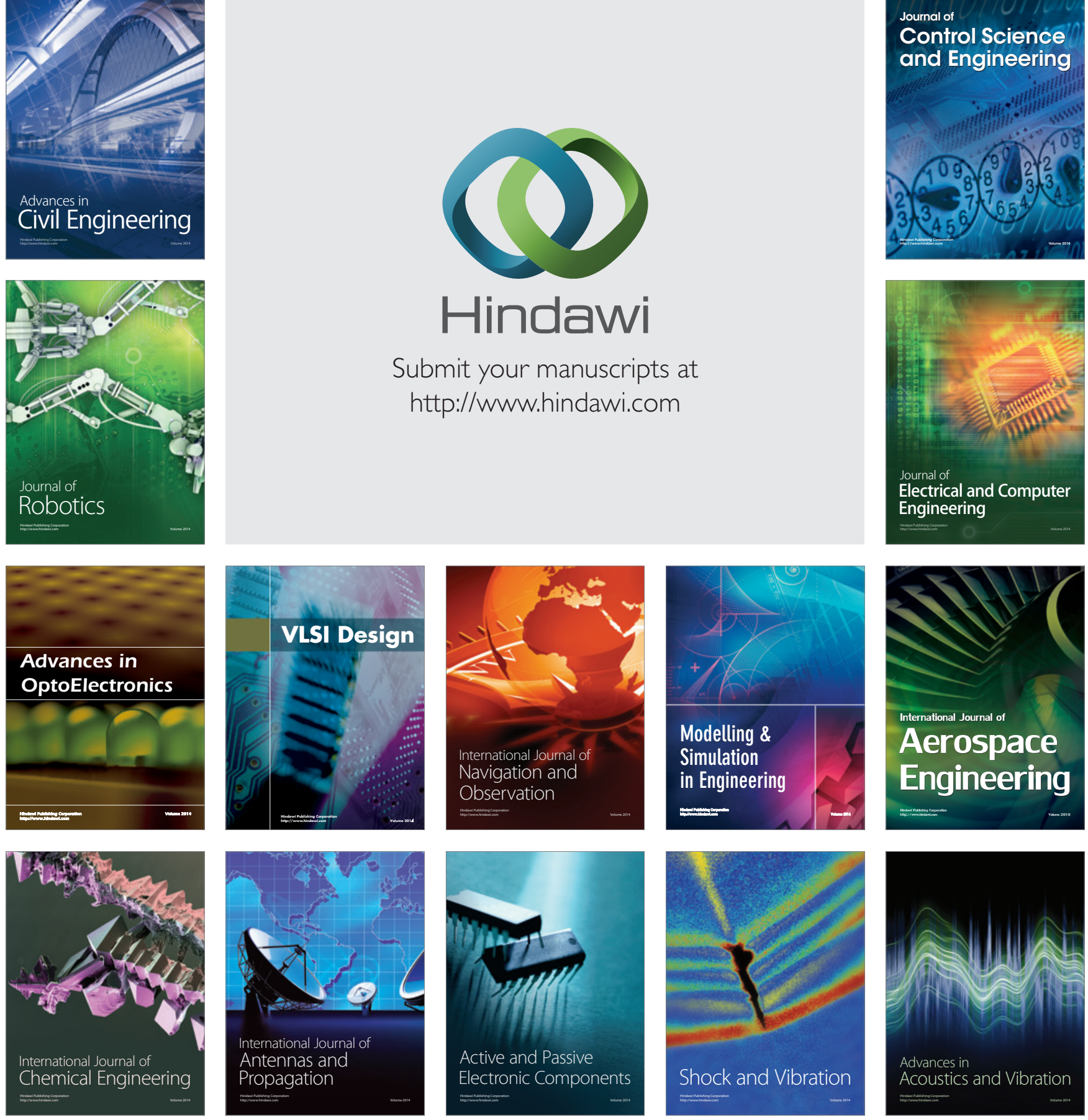